\title{
e-Phaïstos
}

e-Phaïstos

Revue d'histoire des techniques / Journal of the history

of technology

IX-1 | 2021

Autour de Léonard de Vinci

\section{Les « artifices de feu »}

La guerre de siège au milieu du XVI ${ }^{\mathrm{e}}$ siècle

"Fireworks" in Siege Warfare in the Mid-Sixteenth Century Europe

\section{Hugues Paucot}

\section{(2) OpenEdition}

1 Journals

Édition électronique

URL : https://journals.openedition.org/ephaistos/8939

DOI : 10.4000/ephaistos.8939

ISSN : 2552-0741

Éditeur

IHMC - Institut d'histoire moderne et contemporaine (UMR 8066)

Référence électronique

Hugues Paucot, "Les « artifices de feu » », e-Phaïstos [En ligne], IX-1 | 2021, mis en ligne le 30 avril 2021, consulté le 17 septembre 2021. URL : http://journals.openedition.org/ephaistos/8939; DOI : https://doi.org/10.4000/ephaistos.8939

Ce document a été généré automatiquement le 17 septembre 2021.

Tous droits réservés 


\title{
Les « artifices de feu »
}

\author{
La guerre de siège au milieu du XVI ${ }^{\mathrm{e}}$ siècle \\ "Fireworks" in Siege Warfare in the Mid-Sixteenth Century Europe
}

\author{
Hugues Paucot
}

Que Nadine Béague, Philippe Bragard, Philippe Chareyre et Marie Lexa trouvent ici l'expression de ma reconnaissance pour le prêt de matériel, les informations partagées et les conseils prodigués.

1 Développé puis régulièrement utilisé par les Grecs du Bas-Empire contre les Arabes, le feu grégeois constituait probablement la première et la plus connue des armes incendiaires. Ses formules, tenues secrètes pendant quatre siècles, seront finalement révélées par les auteurs arabes de l'époque des croisades, puis par divers auteurs après eux, de Marcus Graecus ${ }^{1}$ à Léonard de Vinci (Berthelot 1891: 787 et 811). Mais cette technologie, en tant que telle, avait déjà disparu des champs de bataille à l'époque de Léonard, l'artillerie à poudre s'étant avérée beaucoup plus efficace. Cette disparition n'était cependant que l'aboutissement de son perfectionnement, qui avait conduit à l'apparition de la poudre elle-même, mais aussi à celle des «artifices de feu ». Si les différents manuscrits de Léonard de Vinci qui nous sont parvenus semblent muets sur ce sujet spécifique (Brioist 2013), ce ne sera pas le cas dans les années qui suivent son décès, où divers traités les décrivent, comme ceux de Battista Della Valle, imprimés pour la première fois en italien en 1521 et traduit dès 1529 en français, ou dans celui de Biringuccio, daté de 1540 et traduit dès 1556 . Tous témoignent en effet de la réalisation, voire de l'utilisation d'artifices incendiaires et explosifs, lors des guerres du XVI ${ }^{e}$ siècle, comme, par exemple, lors du siège de Metz en 1552. En ce qui concerne leur étude, s'il en existe de nombreuses sur les feux grégeois ${ }^{2}$, beaucoup plus rares par contre sont celles qui concernent effectivement les " artifices de feu ». À titre anecdotique, seules quelques publications modernes évoquent brièvement le sujet. Ainsi Reinaud et Favé (1845) parlent des pots et autres lances à feu. Berthelot analysant le Bellifortis, ouvrage médiéval de Konrad Kyeser, avait illustré les flèches et pétards incendiaires, signalant au passage l'usage du chlorure de mercure, désigné sous la dénomination alchimique de servus fugitivus (Berthelot 1900 : 394); et enfin, Fino consacre pour chaque période de 
l'histoire médiévale un petit chapitre aux «feux de guerre», mais sans vraiment s'étendre sur les artifices (Fino 1977).

2 Toutefois, ni les différents traités, ni les publications récentes ne décrivent autrement que grossièrement la conception des armes en question, restant toujours relativement imprécis, voire confus sur les matières et les matériaux utilisés pour leur conception. Or, à ce jour, aucune étude de leur fabrication, de leur mode de fonctionnement et de leur mise en oeuvre n'a jamais vraiment été entreprise.

Pour tenter d'appréhender les matériels et méthodes utilisés pour leur réalisation, ainsi que les effets produits lors de l'usage, plusieurs sources peuvent être exploitées. D'une part, l'étude de plusieurs documents manuscrits, dont un extrait de comptes qui correspond donc à une liste de fournitures, intitulé « Parties et sommes de deniers payées baillées et délivrées comptant [...] pour achapt de plus(ieu)rs drogues et matières qui ont esté néces(sair)es pour la compo(sitio)n des artiffices de feu que led(it) seigneur Duc de Guise a ordonné au Seigneur de S(ain)t Rémy faire pour la tuition ${ }^{3}$ et défense de ceste d(ite) ville de $M e t z^{4} »$, lequel va nous permettre de récupérer des informations détaillées sur les composés et matériaux nécessaires à leur fabrication. Un autre document, listant les armes prêtes à l'usage, intitulé "Inventaire des artifices à feu et autres inventions tant de bois que de fer nécessaires pour la défense de la bresche à Metz inventéz par le S(ieu)r de Sainct Remy de l'ordonnance et commandemant de monseigneur le Duc de Guyse ${ }^{5}$ ", sera également dépouillé pour énumérer les armes préparées lors de ce siège. Les descriptions données par les traités permettront finalement de réaliser la synthèse, en assemblant les pièces du puzzle.

4 Le but de cet article, en proposant une confrontation de ces différents textes, puis une identification des composés mis en œuvre et une étude des mécanismes physicochimiques fondamentaux, est d'établir l'état des connaissances d'un ingénieur français, en l'occurrence Saint-Rémy, au milieu du XVI ${ }^{\mathrm{e}}$ siècle, par rapport à un type d'armes que nous pouvons presque qualifier, pour l'époque, de chimique.

\section{Contexte historique}

Jean de Renaud de Saint Rémy (de Provence) est l'un des rares ingénieurs militaires français ayant acquis une certaine notoriété, au milieu de nombre d'ingénieurs italiens, actifs dans le domaine des fortifications sous François I $^{\text {er }}$ puis Henri II. Bien que sa jeunesse nous soit mal connue, il semble qu'il fit ses classes au cours des septième et huitièmes guerres d'Italie, notamment aux côtés de Pedro Navarro, ingénieur espagnol passé au service des souverains français, et à qui on attribue souvent la paternité des mines explosives. Cet apprentissage est décrit par Brantôme, qui cite Saint Rémy et précise : «je crois que c'est ce brave et vaillant Sainct Rémy qui despuis s'est faict signaler en nos guerres estrangères aux sièges et aux mines et fortiffications, pour l'avoir apris de ce temps de dom Pedro de Navarre (de Bourdeille 1864 : 237) ». Il participe ensuite à la défense de la Provence lors de son invasion en 1536 par les troupes impériales (du Bellay 1912 : 255) puis combat en Piémont, qu'il quitte, semble-t-il définitivement, à la fin de l'année suivante (Marichal 1905: 276). Il s'agit donc à l'origine d'un capitaine, devenu commissaire de l'artillerie, qui se spécialise finalement dans la conception de fortifications bastionnées et participe, entre autres, à divers travaux à Lyon, Guise, Doullens, Hesdin, Montreuil, Bourg-en-Bresse, Toulon, Antibes et Narbonne (Buisseret $2000: 22)$. 
Concepteur des remparts de Saint-Paul-de-Vence (Paucot, Faucherre $2014: 154)$, il est l'un des cent gentilshommes de la maison du roi, et disparait peu après 1557 après avoir, vraisemblablement, été fait prisonnier par les impériaux lors du siège de la ville de Saint-Quentin. Une chapelle funéraire, probable cénotaphe, sise en la collégiale Saint-Martin à Saint-Rémy de Provence, rappelle sa mémoire. Extrêmement sollicité par les souverains français, il est ainsi envoyé en 1552 aux côtés de Charles de Lorraine, duc de Guise, pour la défense de Metz assiégée par Charles Quint en personne. Mais Saint Rémy est aussi le parangon dont se servent nombre de dignitaires français en pleine vague de xénophobie anti-italienne, et qui ne cessent de louer ses mérites, comme par exemple dans les mémoires du maréchal de Vieilleville parlant de ce siège où " le sieur de Saint Rémy, gentilhomme français, natif de Provence, y estoit, et en répputation d'estre le plus suffisant ingénieur en matière de fortifications, et d'admirables inventions d'artifices de feu, qu'on eust sceu trouver en toute l'Europe (Petitot 1822:19)». Mais sorti de ces louanges flatteuses, parfois sujettes à caution pour la raison évoquée ci-dessus, aucun document d'archives n'a jusqu'ici été mis en exergue qui développe réellement les compétences techniques d'un ingénieur comme Saint Rémy en ce qui concerne «les artifices de feu ", ainsi que de leur modus operandi.

\section{L'objet des « artifices de feu »}

7 À l'époque moderne, lors du siège d'une ville ou d'une forteresse, une des techniques les plus fréquentes pour tenter de réduire la place consistait à réaliser une brèche dans l'enceinte soit par l'utilisation de l'artillerie à poudre, soit par la mise en œuvre d'une mine explosive ; puis, dans un second temps, profitant du passage ainsi créé, d'envahir la fortification par un assaut d'infanterie. Pour tenter de colmater la brèche mais surtout d'en interdire l'accès, les défenseurs pouvaient user de divers armements, soit mécaniques (chausse-trape, gabion, pavois ...), soit incendiaire ou explosifs. Parmi ceux-ci, les «artifices de feu» constituent un ensemble d'armement intéressant puisqu'ils font appel à des compétences techniques tout à fait particulières qui font que le détenteur était qualifié, dès cette époque, d'ingénieur, même si sa formation était réalisée principalement par compagnonnage et si aucune université n'avait validé ses compétences. Au siège de Metz, par exemple, divers artifices vont être prévus, dès avant le début du siège, pour faire face en cas de besoin. En synthétisant les documents cités, on peut retenir les items suivants qui constituent une liste des artifices disponibles les plus intéressants : cercle, pot, grenade, pique et lance, le tout « à feu ».

\section{Matières et matériaux}

$\mathrm{Si}$, pour tenter d'en comprendre la fabrication, l'on extrait des documents manuscrits cités ${ }^{6}$, en les classant par catégories, les différents types d'items acquis par l'ingénieur, on peut retenir les produits suivants :

- chanvre long, « fustais », « cordaige », ficelle, corde, grosse cordelle ;

- gros fil de fer;

- « grosses esguilles » ;

- «potzà feu », cercles;

- «cloudz », «cloudz gros et menuz », et «cloudz de grenier »";

- du fil d'achal", " pour employer à lyer les cercles à feu »; 
- des «fueilles de fer blanc ${ }^{10}$ et d'icelle faict canons pour (ser)vir à lances à feu»;

- deux « poinssons d'acier pour percer lesd(it) potz à feu de grenade »;

- de la « toille [...] pour employer à faire grenades, pots à feu, cercles à feu, cartouches ».

Outre ces matériels divers, quelques produits alimentaires ou domestiques communs rentrent également dans les compositions, parmi lesquels on trouve du « viel oing $^{11}$ ", de l'« huylle de lin ${ }^{12}$ » et de l'« huylle dollifi" ${ }^{13}$.

Enfin, de véritables produits «chimiques» sont également cités, parmi lesquels de nombreux dérivés de gemme de résineux, mais également quelques produits d'extraction minière ou sourcé : "godran $»^{14}$, poix molle, "rozine du poix », poix noire, térébentine, « rézine », poix résine, « souffre, pouldre »; ainsi que d'autres provenant plus spécifiquement de chez un "appothicaire»: camphre, "huille de pétrolle», sublimé, "salmoniac ». Les appellations étant parfois nébuleuses, il nous a paru utile de définir précisément ces produits tels que nous les retrouverons plus loin. Certaines appellations étant fluctuantes en fonction du lieu, de l'époque ou des documents, en particulier pour tous les dérivés de la résine de conifère ${ }^{15}$, nous nous sommes efforcés de définir les choses de manière la plus complète possible, en faisant la synthèse de divers sites internet généralistes, et de divers ouvrages imprimés.

\section{Les dérivés de la résine de pin}

10 La térébenthine («tourmentine ») est une oléorésine obtenue après purification ${ }^{16} \mathrm{de}$ la résine brute des résineux, qui est parfois appelée simplement poix. Elle peut être séparée en deux parties par distillation; l'une, solide et inodore, la colophane (" colophone »), appelée ici poix blanche ou "rosine » ${ }^{17}$ ou " raisine »; l'autre, liquide et odorante, l'essence de térébenthine (souvent raccourci en "térébenthine »), ou, pour cela, parfois appelée poix liquide.

11 La colophane est en réalité un mélange d'acides organiques de la famille des diterpènes à $90 \%$ et de $10 \%$ de substances neutres (ester d'acides gras, térébenthine et hydrocarbures). Il s'agit d'une substance combustible, faiblement inflammable, quasi insoluble dans l'eau. Elle est solide et cassante à température ambiante, ne fond pas, mais ramollit à la chaleur (entre 90 à $110^{\circ} \mathrm{C}$ ), et qui peut être utilisée pour coller et imperméabiliser. Pour la rendre plus malléable, elle peut être additionnée d'un autre composé : "Après vous fondrez en un vaisseau, poix raisine avec un petit de gresse ou de terebentine, en sorte que la poix raisine ne rompe (Anonyme $1561: 28 \mathrm{v}^{\circ}$ ) ", ou dissoute dans l'éthanol (donc dans l'eau-de-vie dont il sera question plus loin) ou dans l'essence de térébenthine.

12 L'essence de térébenthine est un mélange comprenant en particulier des composés terpéniques, des acides et des alcools. Ce liquide est insoluble dans l'eau et hautement inflammable ; son extinction ne pourra dès lors être réalisé que par étouffement.

13 Le goudron est produit par la pyrolyse du bois, souvent de résineux épuisés par le gemmage, lors de la fabrication de charbon (Cauvet $1885: 122$ ), ou par simple cuisson de la résine (Aubin 1702 : 463). Il est constitué essentiellement d'oléorésine altérée par la chaleur et noircie par le noir de fumée. Utilisé principalement pour le calfatage des navires, il peut être séparé en l'huile de poix et en poix molle, laquelle, après séchage à chaud, devient cassante et collante une fois refroidie ; selon les procédés de fabrication, on parle aussi de poix navale ou de poix noire. 
Enfin, pour en finir avec les composés issus des résineux, la poix résine est de la rosine, dans laquelle, pendant une simple fusion, on a réussi à introduire au moyen du brassage une certaine quantité d'eau. Elle était commercialisée sous forme de pains de couleur jaune, presque totalement opaques, très fragiles, se ramollissant à la chaleur des mains (Pelouze 1839 : 396).

\section{L'huile de pétrole}

Localement, il s'agit vraisemblablement d'huile issue de la source dite Baechel-Brunn à Lampertsloch (67), et signalée dès 1498 par Wimpfeling (de Chambrier 1919: 2). Elle produisait une sorte de bitume dont on se servait pour lubrifier les moyeux des roues des chariots et comme produit de soin à usage externe (Chambrier 1919: 3). D'un point de vue chimique, il est constitué d'un mélange de molécules d'hydrocarbures plus ou moins longues, et donc plus ou moins volatiles et inflammables. Saint-Rémy devait avoir appris l'usage de l'huile dans la fabrication des artifices en Italie, puisque, dès 1460, François Arioste vante au duc de Modène les propriétés d'une source de pétrole découverte au Mont-Gibbuis, près de cette ville (Bouvet 1939 : 5).

\section{Le camphre}

16 Le camphre est une cétone (1,7,7-triméthylbicyclo [2,2,1]-heptan-2-one) préparé en distillant, avec de l'eau, le bois du camphrier (Laurus camphora). C'est un solide blanc, quasi insoluble dans l'eau, mais soluble en phase organique ; il est également volatil et combustible. Le risque d'explosion de poussières dans le cas de poudre ou de granulés mélangés à l'air est important; de plus, il réagit violemment avec les oxydants puissants, les réducteurs puissants et les solvants chlorés ${ }^{18}$.

\section{Le sublimé (corrosif)}

Il s'agit en réalité du chlorure mercurique $\left(\mathrm{HgCl}_{2}\right)$, employé autrefois comme antiseptique, notamment pour la syphilis. Sa synthèse résulte d'une série de réactions au départ de $\mathrm{Hg}$ ( « vif argent») et de $\mathrm{HNO}_{3}$ (« esprit de nitre ), puis de $\mathrm{FeSO}_{4}$ (« vitrol calciné ») et de $\mathrm{NaCl}$ (Bourzat 2005 : 116). Dans le cas du siège de Metz, il ne nous a pas été possible de déterminer s'il s'agissait d'une production locale, réalisée par l'apothicaire lui-même, ou d'un produit d'importation. Mélangé avec du soufre et du carbone, il réagit avec le soufre, quand bien même la thermodynamique ne serait pas favorable à cette réaction, catalysée par la présence du carbone, comme l'ont constaté Ren et Zhu (2015: 822) :

$$
\mathrm{HgCl}_{2}+\mathrm{S} \rightarrow \mathrm{HgS}+\mathrm{Cl}_{2}
$$

18 Le sublimé sert donc ici de source de mercure, lequel est fréquemment utilisé dans la préparation des artifices et souvent mélangé au soufre avec lequel il tend spontanément à reformer HgS par des liaisons covalentes.

\section{"Salmoniac ", " sal ammoniac », " salmiac », « selarmonial » ou encore " sel armoniac »}

Le "sel Armoniac" des Anciens «n'était autre chose que le sel volatil de l'urine des chameaux et de plusieurs autres animaux qui passaient en grand nombre par des pays forts chauds, comme 
par les déserts de la Libye, par l'Arabie. L'urine de ces animaux était consommée, peu de temps après avoir été faite, par la grande ardeur du soleil et l'on trouvait son sel volatil sublimé à la superficie des sables (Bourzat 2005 : 147) ». En fait, il s'agit de chlorure d'ammonium $\left(\mathrm{NH}_{4} \mathrm{Cl}\right)$ qui se décompose lors de chauffage, produisant des fumées toxiques et irritantes (oxydes d'azote, ammoniaque et chlorure d'hydrogène). Même si les mémoires de l'Académie des sciences témoignent des interrogations que ce sel suscitait encore au XVIII ${ }^{e}$ siècle, il semble que sa synthèse était relativement simple à réaliser, puisqu'on mettait à "sublimer dans des ballons de verre, avec un peu de sel marin et d'urine, la suie provenant de la fiente des bestiaux» (Bourzat 2005 : 149).

Toutefois, rien ne nous permet d'affirmer que le processus de synthèse était déjà connu dans l'est de la France au milieu du XVI ${ }^{\mathrm{e}}$ siècle, et qu'il ne s'agissait pas alors d'un produit d'importation, provenant principalement d'Égypte. En phase aqueuse, mélangé au salpêtre (nitrate de potassium), on observe rapidement l'équilibre suivant :

$$
\mathrm{KNO}_{3}+\mathrm{NH}_{4} \mathrm{Cl} \leftrightarrows \mathrm{KCl}+\mathrm{NH}_{4} \mathrm{NO}_{3}
$$

produisant du nitrate d'ammonium, oxydant puissant et explosif particulièrement violent ${ }^{19}$. Plusieurs mécanismes de décomposition ont été proposés, parmi lesquels celui-ci est le plus exothermique (Cagnina $2014: 26$ ) :

$$
2 \mathrm{NH}_{4} \mathrm{NO}_{3} \rightarrow 2 \mathrm{~N}_{2}+4 \mathrm{H}_{2} \mathrm{O}+\mathrm{O}_{2}
$$

Ceci étant, il est normalement insensible aux chocs et aux frottements, sauf s'il est mélangé à des combustibles comme des hydrocarbures, ou s'il est fondu et confiné lors, par exemple, d'un incendie violent. De plus, parmi les incompatibilités connues du nitrate d'ammonium, l'une des plus importantes est certainement celle concernant les espèces chlorées et notamment des sels comme le chlorure d'ammonium et la chlorure de sodium ( $\mathrm{NaCl}$ ) (Cagnina $2014: 30$ ). Nous verrons plus loin que la mise en œuvre des différentes huiles constitue donc un catalyseur important, et/ou que le salmoniac, mais également le chlorure de sodium, pouvait en plus également jouer ce rôle.

$\mathrm{Au}$ final, le nombre de fournisseurs différents intervenant pour ces produits, parfois pour un même item, et l'absence de chiffres ronds dans les volumes commandés, indiquent sans doute que l'ingénieur était contraint de faire l'acquisition de la totalité $\mathrm{du}$ stock de chacun des fournisseurs. De plus, certains des produits liquides inflammables sont, compte tenu de leurs propriétés physiques, a priori interchangeables, ce qui permettait d'accroître les quantités acquises à effet similaire. Ainsi, la térébenthine et l'huile de pétrole ont des valeurs d'inflammabilité et d'explosivité similaires, de même que l'huile d'olive et de lin (tableau 1). Si les quantités disponibles sur le marché s'avéraient limitées, on pourrait ainsi expliquer la variété des produits achetés. On peut aussi raisonnablement penser, que compte tenu des filières d'approvisionnement, Saint-Rémy a dû s'adapter à la géographie, apprendre à utiliser l'huile d'olive lors de ses campagnes italiennes et trouver un succédané en Lorraine sous la forme de l'huile de lin. Il est intéressant de remarquer que le salpêtre, souvent évoqué ci-dessous, n'apparaît pas dans la liste des fournitures. Comme pour la poudre et le soufre, les besoins, en temps de guerre, sont tels que les fournisseurs habituels aux armées devaient pourvoir à son approvisionnement. 
Tableau 1 : Propriétés physiques des produits liquides cités

\begin{tabular}{|l|l|l|l|}
\hline Dénomination & $\mathrm{T}^{\circ}$ d'ébullition $\left({ }^{\circ} \mathrm{C}\right)$ & $\mathrm{T}^{\circ}$ d'auto-inflammation $\left({ }^{\circ} \mathrm{C}\right)$ & Point d'éclair $\left({ }^{\circ} \mathrm{C}\right)$ \\
\hline Essence de Térébenthine & $154-170$ & 220 & 35 \\
\hline $\begin{array}{l}\text { Huile de pétrole } \\
\text { (Pétrole lampant) }\end{array}$ & $250-285$ & $>220$ & $>38$ \\
\hline Eau de vie (éthanol à 40-50 \%) & 79 & $423-425$ & $21(70 \%$ vol.) \\
\hline Huile d'olive & 300 & 343 & 225 \\
\hline Huile de lin & 316 & 225 & 110 \\
\hline
\end{tabular}

Les valeurs des propriétés physiques des produits liquides évoqués dans le texte et présentées ici sont données à titre indicatif ; elles devaient en effet certainement présenter des différences entre la valeur théorique et la réalité du terrain, en fonction de la pureté, de la composition exacte, etc.

\section{Réalisation et principes de fonctionnement}

En synthétisant, on arrive à la description des armes suivantes :

\section{Le cercle de feu}

Pour nous aider dans la compréhension de la réalisation et du mode de fonctionnement $\mathrm{du}$ dispositif en question, nous pouvons avoir recours à la description assez précise donnée en 1598 par Boillot :

«Le cercle à feu pour jetter sur les troupes à un assault est ainsi faict: vous prendrez cercles desquels on relie les tonneaux les tremperez en poix fondue \& pouldre à canon, mestez ensemble, puis prenez de la toille aussi long que le cercle de tour [...] Puis prenez les mixtions faictes d'une livre de grosse pouldre, une once de soulfre, trois livres de salpestre mettez à part chascune partie en poudre. Puis les meslez ensemble à la main, avec un peu d'huille de lin [...] Puis vous accomoderez un autre pareil cercle de semblable façon les croiserez \& lierez de fil de fer si bien qu'ils ne se puissent serrer, ou séparer quand on les jettera, les voulant jetter sur l'ennemy, mettez le feu en l'amorçe que vous avez en celuy qui est couvert de toille, \& le tenez par celuy qui est couver destouppes » (Boillot 1598 :151). 
Fig.1. Cercle de feu, exemple de carcasse incendiaire

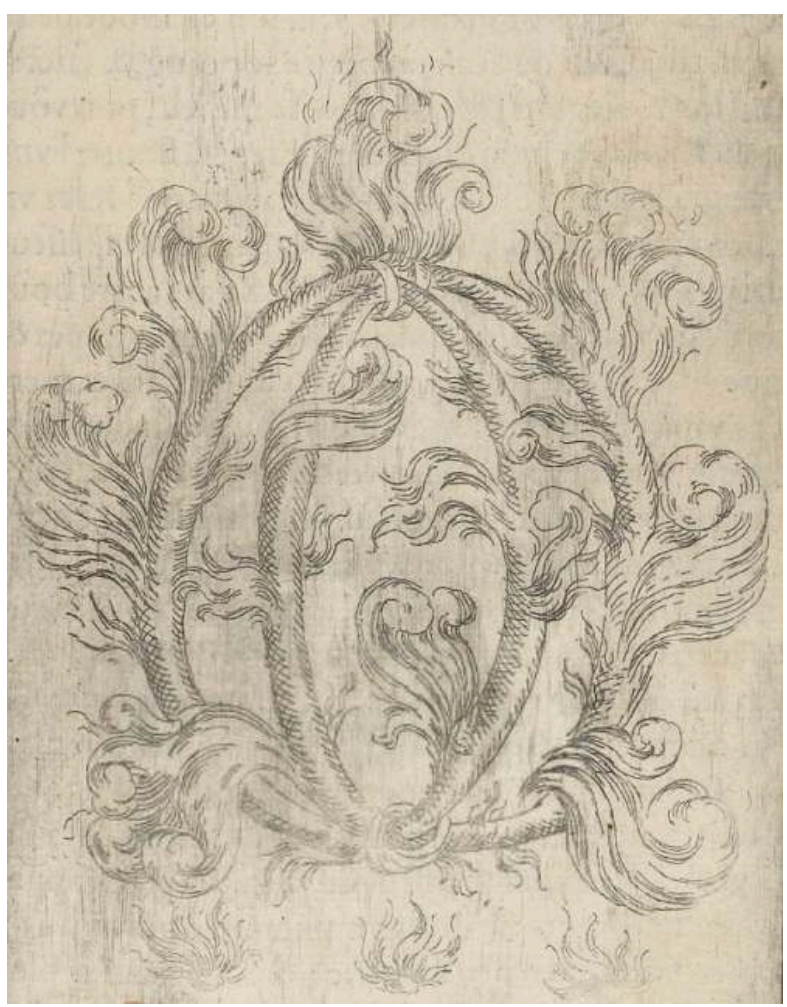

On distingue les deux cercles métalliques liés perpendiculairement et les flammes qui s'échappent des matériaux enrobant les cercles.

Boillot 1598 : 152; Source : gallica.bnf.fr/BnF

Il s'agit donc de deux cercles de tonneaux fixés ensemble perpendiculairement par du fil de fer ou d'archal, et trempés dans un mélange de résine purifée, agglomérée de poudre à canon. Au moins un des cercles est recouvert de toile emballant un mélange de matériaux aux propriétés inflammables (poudre, soufre en poudre ou en bloc, salpêtre), constitués en pâte huileuse par un mélange dans de l'huile de lin qui sert donc ici d'agglomérant tout en participant à la combustion. La poudre étant diluée, le caractère explosif du mélange devait être fortement atténué et s'étaler dans le temps. La combustion, sur tout le pourtour, devait durer plusieurs minutes après l'allumage, du fait de blocs de soufre (« roche de soulfre ») utilisés. Ainsi, les cercles, en roulant vers l'adversaire, devaient pouvoir perturber fortement les vagues d'assaut des assiégeants se ruant vers une brèche. Le document Clairambault 346 précise aussi qu'ils pouvaient être jetés à l'aide de "fourches de fer avec leurs hampes ».

\section{Les pots à feu}

27 Ceux-ci sont également nommément cités à plusieurs reprises dans l'inventaire et la liste d'achat de matériaux. Dans ce cas de figure, où on se place du côté du défenseur, on peut raisonnablement penser qu'il s'agit d'un récipient destiné à être envoyé sur l'adversaire et non d'un engin explosif ayant pour fonction de faire sauter une porte par exemple. Les principes d'élaboration sont exposés à la fois par Della Valle, Biringuccio et par Boillot, mais il est intéressant de se pencher également sur la composition du mélange décrit en 1621 par Diego Ufano. 
Fig.2. Représentation de pots à feu

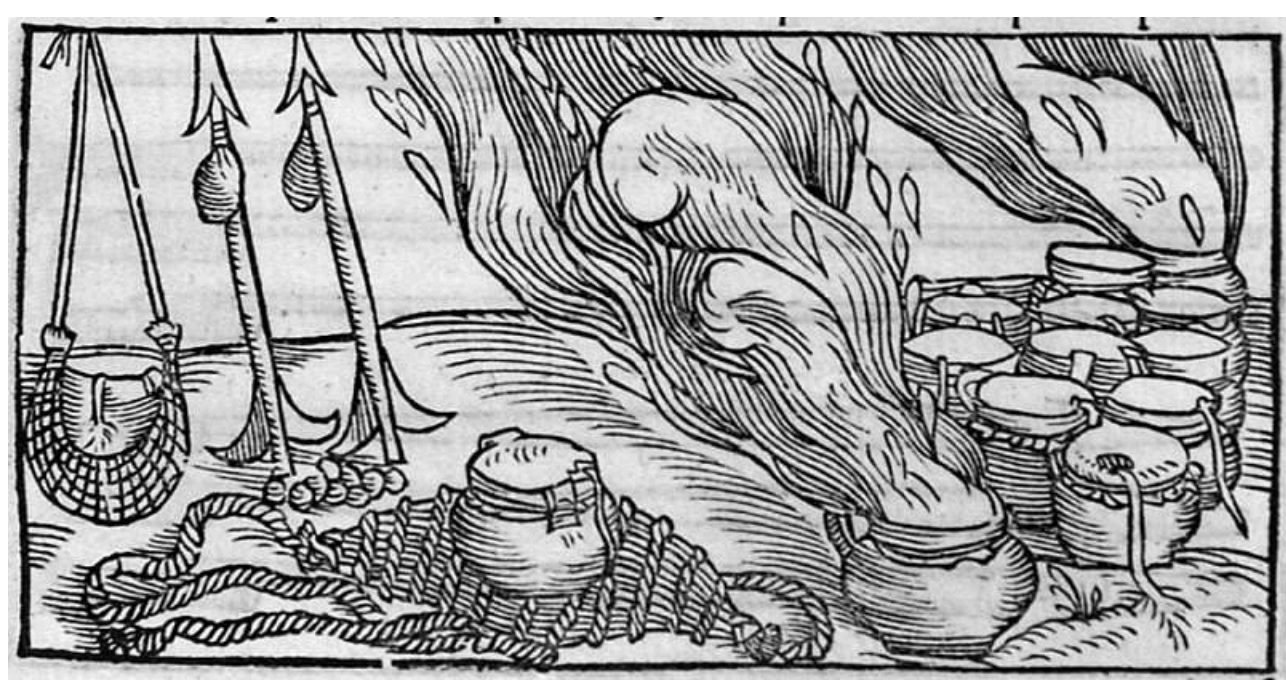

Sur cette gravure, il est possible d'observer les pots à feu et un filet de transport ou de lancement. De même, la fermeture des pots par de la toile imbibée, simplement percée pour permettre la mise en place de la mèche, est également visible.

Biringuccio 1572 : $164 v^{\circ}$; Source : http://www.bvh.univ-tours.fr

Ainsi, la recette la plus ancienne retrouvée est celle de Della Valle. Il évoque un pot en terre, rempli d'un mélange de "poudre de bombarde ${ }^{20}$, deux parts, poix grecque ou poix d'Espagne une part, poix navale ou poix de navire une part. Broye très subtilement tout ensemble dans un mortier, puis aie de la tourmentine en suffisance dans une chaudière, mettre sur le feu, mettre dedans un peu d'huile de lin et si on ne peut en avoir, de l'huile de genévrier ${ }^{21} »$. Cette description simpliste mérite d'être complétée. Pour le mélange explosif, Diego Ufano décrit l'usage du sel ammoniac (chlorure d'ammonium) et du camphre, trouvé dans la liste des matières acquises par SaintRémy :

«Pren de la pouldre, soulfre, salpêtre, sel armoniac, de chascun $1 / 2$ $\mathrm{Lb}^{22}$. Camphre 2 once, le tout bien molu \& estamisé, adjoutte y une poignée de sel commun $(\mathrm{NaCl})$, mets le tour ensemble en une payelle ou petit chauderon, avec aultant de poix liquide, ou huyle petrolis, ou de lin, ou de noix qu'il se face une paste aucunement dure " (Ufano $1621: 126)$.

Une autre description donnée par Biringuccio permet de compléter la recette :

\begin{abstract}
«Et se font ces pots de terre feiche. Puis on met dedans de grosse poudre meslée avec poix grégoise \& soufre brisé. Puis on met audessus gresse de pourceau pour couvrir la poudre, \& l'incorpore on dedans icelle l'épesseur d'un doigt, afin que la poudre ne vienne à se respandre, \& que le feu s'yi empoigne plus lentement, jusques à ce qu'il soit arrivé auprès des ennemis » (Biringuccio $1572: 164$ vº).
\end{abstract}

Si la « graisse de pourceau » fait incontestablement référence au «viel oing » décrit plus haut, on peut par contre s'interroger sur la " poix grecque ou grégoise». On peut retenir ici la composition donnée par Léonard de Vinci, à savoir " prenez charbon de Saule, nitre ${ }^{23}$, eau-de-vie, résine, souffre, poix, camphre» (Venturi 1797:30). Une autre recette plus 
complexe est décrite en 1561 (Anonyme 1561:54 vº), mais elle est en réalité copiée sur Marcus Graecus. Elle comporte du «souffre vif, tarte ${ }^{24}$, farcacoly ${ }^{25}$, peghel ${ }^{26}$, sarcofti $i^{27}$, olei petroli $i^{28}$, olei co $(\mathrm{m}) \mathrm{min}^{29}$. Et faictes bien bouillir lesdictes matières auecques gomme dragaga $(n) t^{30} »$. Cette description devait permettre d'obtenir un produit gélatineux, la gomme du tragacanthe assurant la cohésion de l'ensemble des composés, la plupart combustibles. Notons que ni le tartre, ni la résine de sarcocollier, ni la gomme n'apparaissent dans les produits utilisés par Saint-Rémy, contrairement à l'huile de pétrole et à la poix.

31 Cependant, quelle que soit la mixture considérée, le mélange des composés, lié à l'huile de lin, ou autre, devait avoir une texture pâteuse. Il ne pouvait être introduit que dans un pot possédant un goulot suffisamment large, même s'il était spécifié "estroict à la bouche» (Boillot 1598: 157) ou "que la bouche ne soit non plus grande que pour mettre le

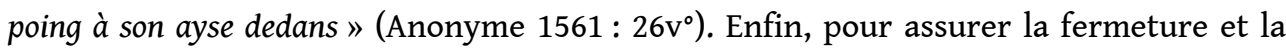
conservation de l'ensemble en attente d'usage, il était conseillé de « couvrirez vostre pot de toille avec poix résine fondue » (Boillot 1598 : 157). Ce «couvercle» est parfaitement visible sur la figure 2 .

Quoi qu'il en soit, l'usage de ces pots ne pouvait se faire que par jet quasi vertical au pied de la courtine ${ }^{31}$, comme en témoigne Paolo Giovio lors du siège d'Albe Royale ${ }^{32}$ en 1562 par les Turcs, et qui dévoile également la parade :

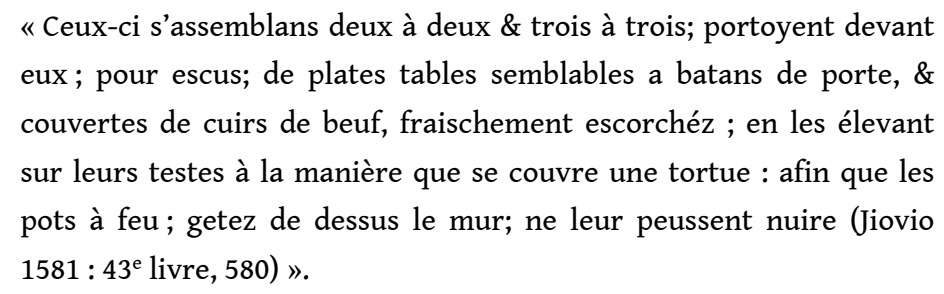

\section{Les grenades} pouvaient être réalisées de deux manières, soit en utilisant un petit pot de terre cuite, cette technologie étant un héritage des Byzantins, soit en utilisant des petits sacs de toile comme contenant. Il apparaît que, d'après les manuscrits, dans le cas du siège de Metz, Saint-Rémy a adopté les deux techniques.

En ce qui concerne le sac de toile, Della Valle décrit des balles, ou boules de feu, qui peuvent s'y apparenter :

«Aie cinq parts de poudre d'artillerie lourde, trois parts de salpêtre raffiné, deux parts de soufre, une part de poix raisine, une demi-part de camphre, une demi-part de tourmentine, une demi-part de voirre $^{33}$ pilé, pas trop, une demi-part de sel commun ${ }^{34}$, une demi-part d'huile de roche ${ }^{35}$ et d'huile de lin, c'est-à-dire en part égale, et demipart d'eau-de-vie, et toutes ces choses soient bien mélangées ensemble, ensuite prends une pièce de toile quand tu veux faire la balle et la faire en manière de bourse, et la remplir de ladite mixture ${ }^{36}$ ». 
si les listes de matériels de Saint-Rémy n'en parlent pas, on peut cependant s'interroger sur l'ajout du verre pillé dans le mélange, dont il sera encore question en 1741 (Saint Rémy 1741 : 349). Siemienowicz apporte sans doute une explication sur sa présence en précisant ceci :

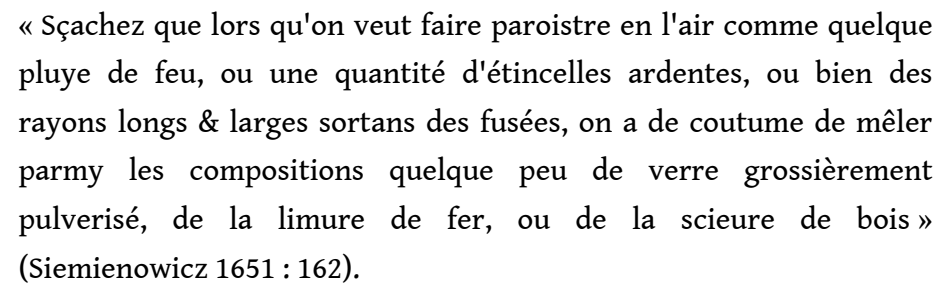
pluye de feu, ou une quantité d'étincelles ardentes, ou bien des rayons longs \& larges sortans des fusées, on a de coutume de mêler parmy les compositions quelque peu de verre grossièrement pulverisé, de la limure de fer, ou de la scieure de bois" (Siemienowicz $1651: 162$ ).

La projection de gouttes de verre en fusion devait donc être potentiellement blessante, mais leur aspect incandescent devait aussi être propre à rendre la combustion encore plus impressionnante.

Pour ce type de grenade, nous pouvons aussi nous référer à l'ingénieur anonyme de 1561 qui donne une description assez explicite (Anonyme 1561:28 vº). En résumé, dans un double sac de toile, on constitue un mélange des mêmes composés (soufre, poudre non grenée, salpêtre) mais additionné de camphre et de "vif argent ", "puis méslez tout ensemble à la main avec un petit d'huile pétrole, \& en faute de ladite huile mettez huile de lin " Le tout est ensuite recouvert de "poix raisine [fondue] avec un petit de gresse ou deterebe(n)tine, en sorte que la poix raisine ne rompe, quand vostre po $(m)$ me sera faicte ». Dans ce dispositif, la présence du mercure est intéressante. D'après Reinaud et Favé, parlant de la poudre des Chinois: «si l'on veut une explosion forte, [...] on y ajoute encore du mercure » (1845: 179). Comme explicité précédemment, Saint-Rémy utilisait le sublimé comme source de mercure, lequel va se recombiner au soufre pour former HgS qui peut alors réagir violemment avec les agents oxydants puissants, comme le salpêtre, donnant de quoi envenimer encore la réaction. L'auteur décrit aussi ici l'usage des aiguilles à coudre (les sacs) et des poinçons (pour l'introduction de la poudre d'amorçage) listés dans le matériel nécessaire.

L'autre technique utilisée pour réaliser les grenades faisait appel à des petits pots de terre cuite. Il semble que ceux-ci aient également été prévus, puisque dans un autre passage d'un des documents manuscrits cités, il est spécifié que les pots devaient " être percés par [...] deux poinssons d'acier pour percer lesd(it) potz à feu de grenade [...] ${ }^{37}$ ». 


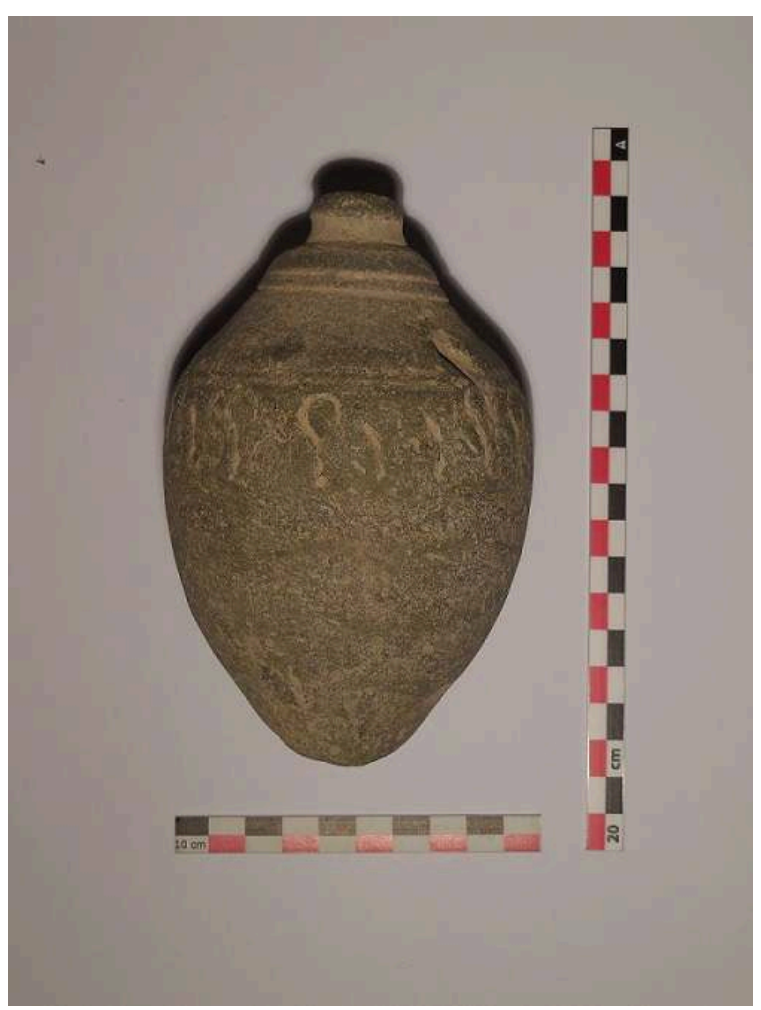

La grenade présentée ici, typique de la fin du premier millénaire (ca. 800-1200), illustre ce type de dispositif réalisé dans un pot en terre. L'orifice d'entrée de $7 \mathrm{~mm}$ de diamètre n'autorise donc son remplissage que par un liquide ou une poudre fine. Son poids de $720 \mathrm{gr}$. permettait un lancer manuel ou, plus vraisemblablement, à l'aide d'une fronde.

Collection particulière. Photographie de l'auteur

Dans ce cas, les préparations décrites ci-dessus ne peuvent être utilisées, car formant des pâtes trop denses voire solides, et par conséquent difficiles à introduire par l'orifice des grenades, large au maximum d'une dizaine de millimètres. La photographie de la grenade présentée ici (fig.3) illustre bien la difficulté qu'il y aurait eu à remplir le volume intérieur avec la mixture décrite précédemment. La matière inflammable ne pouvait donc être constituée que d'une poudre fine, d'un liquide, ou, comme Biringuccio le décrit ci-dessous, d'un mélange solide chauffé pour le liquéfier. Notons au passage que Della Valle donne une composition assez proche, mais d'un mélange qui apparaît fort solide, et donc a priori, trop imprécis pour pouvoir effectivement être utilisé dans ce cas précis (Della Valle 1529: $10 \mathrm{r}^{\circ}$ ). Pour Briniguccio, par contre, "encores fait-on une autre composition liquide \& coulante dans une chaudière, en laquelle faut mettre gresse de pourceau, huille de soufre vif, salpêtre deux fois réafiné, eau-de-vie, poix grégoise, tourmentine, \& quelque quantité de grosse poudre. Et le tout ayant esté réduit en liqueur, remurez la matière avec un basto(n), puis en re(m)plirez les pots à demi. Et au-dessus, mettrez qualité de pouldre, afin que le feu se puisse prendre plus facilement. Et quand vous les voudrez mettre en oeuvre, vous les jetterez avec une $f(r)$ onde (Biringuccio, $1572,164 v^{\circ}$ )».

En ce qui concerne l'usage du poinçon et le perçage du «potz à feu de grenade », cette manipulation devait sans doute être réalisée juste avant l'usage pour y introduire une mèche. En effet, si les textes sont muets sur l'allumage, il n'est pas non plus question de bouchon éventuel. Le mélange, introduit chaud et liquide, devant se resolidifier à l'intérieur mais la poudre introduite en suivant aurait pu s'humidifier. Il faut donc 
imaginer un petit bouchon réalisé sans doute également avec le mélange fondu. La couche superficielle, éventuellement altérée, aurait alors été crevée avec le poinçon et la mèche introduite jusqu'au cœur de la substance. Deux poinçons auraient alors suffi à équiper les artificiers peu avant l'usage. L'apparition de l'eau de vie (éthanol à 40-50\% vol. environ) est intéressante. Elle joue incontestablement le rôle de produit inflammable, mais aussi de dissolvant ou fluidifiant des graisses ; il faut cependant bien maîtriser la température de préparation pour faire fondre la graisse de porc $\left(30-35^{\circ} \mathrm{C}\right)$ et assurer le remplissage sans pour autant évaporer tout l'éthanol $\left(79^{\circ} \mathrm{C}\right)$.

\section{Les piques à feu}

Les piques à feu constituent un autre artifice intéressant. Une description nous en est une fois encore donnée par le traité anonyme de 1561 dans lequel apparaît qu'il s'agit d'une "pique de neuf à dix piedz $z^{38}$ de long ", enrobée près de son extrémité pointue d'un "sac de toile en double de deux paulmes ${ }^{39}$ de lo(n)g, estroid par les deux boutz, \& large au milieu ", remplis des ingrédients habituels, de mercure, mais aussi de plomb en poudre et de verre battu, agglomérés à l'huile de lin. Le sac est ensuite recouvert de " roche de souffre, de poix noire ou de poix raisine. Et qua(n)d vous vouldrez faire servir vostre picque [...] y mettez le feu, c'est un baston qui est bon à une brèche, ou à autre passage qui est estroit pour se défendre, \& aussi pour assaillir son ennemy » (Anonyme 1561:26 $\mathrm{r}^{\circ}$ ).

Fig.4. Piques ou langue à feu

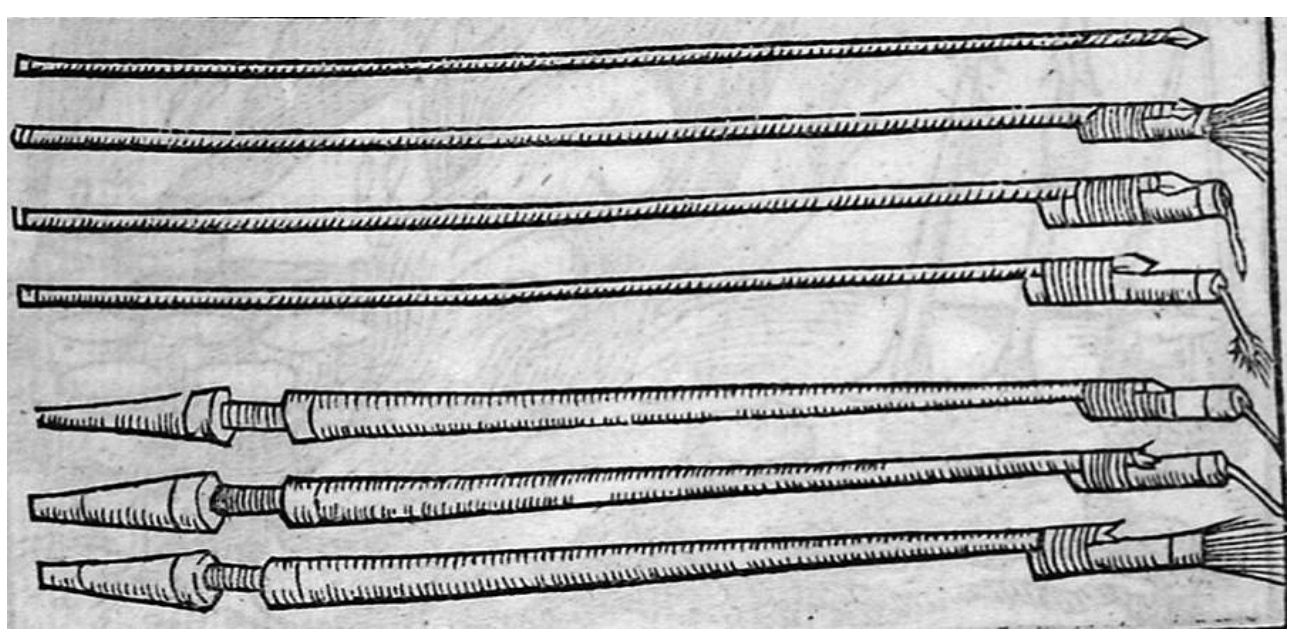

Ce type d'armement ne devait permettre la création d'une flamme que sur un temps relativement court et son interaction avec l'adversaire, qu'à courte distance.

Biringuccio 1572 : 164 r' ; http://www.bvh.univ-tours.fr

Même si les listes de matériels de Saint-Rémy n'en parlent pas, on peut cependant s'interroger sur l'ajout du plomb en poudre $(\mathrm{Pb})$ dans le mélange. À part une coloration de la flamme blanc-bleu ou à imaginer une réaction complexe générant de l'azoture de plomb explosif, il ne semble pas que l'on puisse lui donner un rôle particulier; il disparaît d'ailleurs des recettes dès le siècle suivant, comme dans celle d'Ufano. Enfin, la calcination devait endommager fortement l'extrémité de la lance, laissant l'utilisateur handicapé voire désarmé. On peut donc s'interroger sur la réelle efficacité du dispositif. 


\section{La trompe ou lance à feu}

43

Contrairement à l'idée généralement répandue qui laissait l'exclusivité de l'usage des lances à feu aux utilisateurs chinois, l'usage des feuilles de fer blanc paraît ici relativement claire puisqu'il est dit dans le document: "VIII S(ols) ce pour avoir couppé cinquante fueilles de fer blanc et d'icelle faict canons pour (ser)vir à lances à feu $»^{40}$.

Divers dispositifs peuvent être envisagés comme ayant été potentiellement construits par Saint Rémy. En effet, plusieurs parentés existent dans les descriptions trouvées dans les traités et les matériaux acquis par l'ingénieur. Pour Della Valle (Della Valle 1529) et Biringuccio (Biringuccio $1572: 162 \mathrm{r}^{\circ}$ ), on pourrait considérer les trompes à feu et pour Boillot (Boillot 1598 : 149), les lances à feu.

« Les trompes se pourront faire \& se font encores, de bandes de fer lombard $^{41}$, ou de lames de cuivre. Et pour les faire, on fait un canon de bois long d'une brasse $\&$ demie $^{42}$, lequel on cie par le milieu ${ }^{43}$. [...] Puis au fond, vous ne faudrez à mettre la haulteur de quatre doigts de poudre à canon. Au-dessus de laquelle, vous mettrez un boulet fait $\mathrm{d}^{\prime}$ estoupes ${ }^{44}$ ou de pièces de toille, au milieu de laquelle vous avez mis quantité de poudre. Et au-dessus, vous mettrez pour le couvrir quatre doigts de grosse poudre co(m)posée avec poix grecque, voirre brisé, gros sel commun, salpêtre \& écaille de fer aucuneme(n)t brisée. Et audessus de ceste composition, vous sèmerez deux doigts de poudre fine. Et dessus ce boulet en mettrez un autre fait de la mesme façon » (Biringuccio 1572:162 $\mathrm{r}^{\circ}$ ) et ainsi de suite.

Fig.5. Trompes à feu

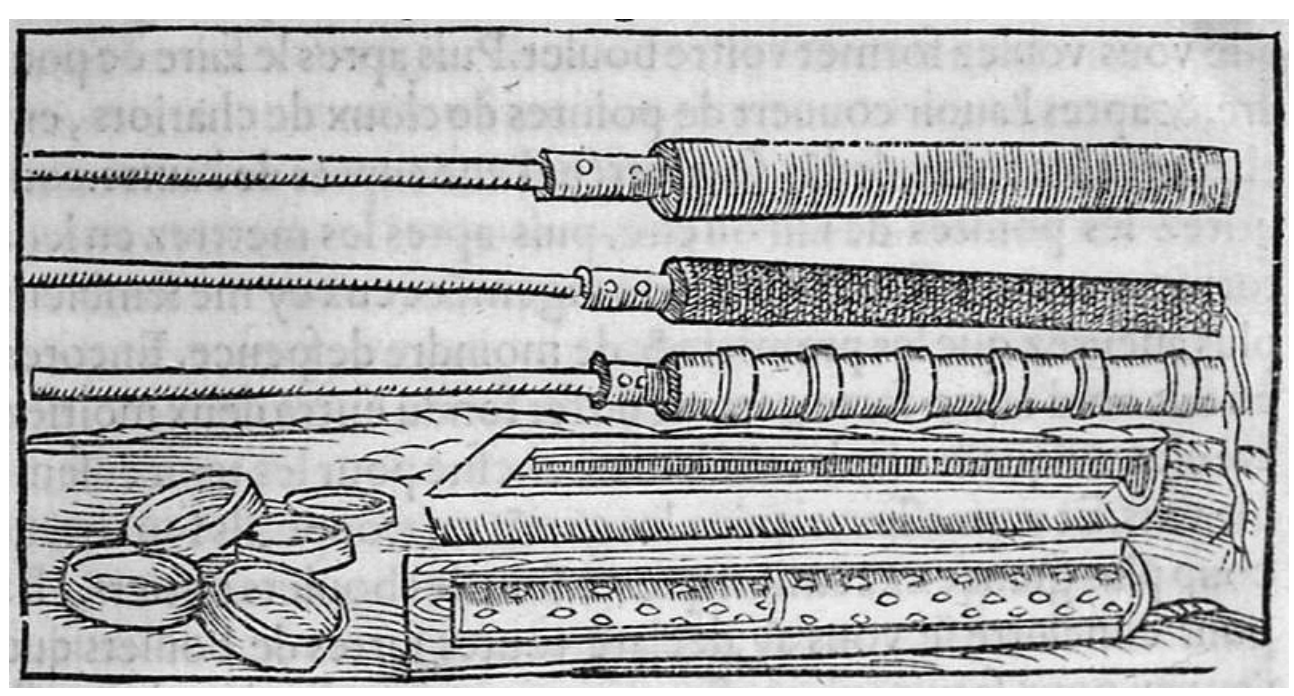

Sur ces représentations de trompes à feu, on distingue dans la partie basse de l'image le tube scié en deux pour permettre son remplissage, la fermeture du dispositif par des cercles, sans doute métallique, ou vraisemblablement par du fil de fer (dans l'exemple du haut), comme décrit dans les documents cités dans le texte.

Biringuccio 1572 : 163 ro; http://www.bvh.univ-tours.fr

On a peine à imaginer comment un tel dispositif, de plusieurs mètres de long, d'un poids non négligeable et totalement déséquilibré, pouvait être porté et manipulé simplement. Par ailleurs, il est extrêmement intéressant de constater que Saint Rémy 
fait réaliser les trompes en fer blanc - vraisemblablement en remplacement ou s'inspirant du fer lombard dont il a été question plus haut - et non pas en bois creusé selon la description proposée par Boillot à la fin du siècle. Il est certain que la conservation du matériel en attente de service devait être meilleure tout en évitant le traitement imperméabilisant à la poix recommandé " pour la garder de pourriture " (Boillot 1598 : 149). De plus, la température de fusion relativement élevée du fer devait permettre une meilleure résistance à la chaleur qu'une enveloppe en bois prompte à s'enflammer. Observons la présence du mercure et sa réactivité déjà mentionnée en présence de soufre. Le mélange avec l'huile de pétrole, constitué d'une variété importante de molécules d'hydrocarbure, laisse donc supposer de possibles réactions, augmentant encore l'inflammabilité de la préparation.

En définitive, l'accumulation, en couches successives, de matériaux inflammables dans le canon de la lance, était supposée la transformer en une espèce de lance-flammes, version Renaissance. Une expérience d'archéologie expérimentale, malheureusement mal documentée, a permis de faire brûler un dispositif similaire pendant 18 à 19 secondes (Smith 2018: 39). Ce type d'armement était aussi utilisé par les assaillants, mais certaines réserves existaient réellement quant à sa mise en oeuvre. Ainsi dans ses Discorsi Militari datés de 1587, Antonio Lupicini précise :

\begin{abstract}
« quant aux trompes à feu, [...] outre l'embarras de les amener, elles trompent souvent à l'usage, notamment dans les assauts où il faut être très accort pour bouter feu à la mèche ; autrement la trompe fuse en l'air ou nuit à ton infanterie ; si même le feu est bouté à temps, nous ne sommes aucunement certains que la composition n'est pas trop sèche, alors elle éclate entre les mains de qui l'exécute ; si elle est trop humide, elle se résout en fumée. Admettons que tout soit à point, chose rare ; comment empêcherons-nous les défenseurs de les renverser avec leurs hallebardes ou de les tourner en arrière, au grand dommage de l'assaillant, comme cela s'est vu aux assauts de Vicovaro ; puis, ce qui est pis, s'il fait un peu de vent venant sur toi, tu as la fumée et les jets de flammes, comme il advint aux Espagnols aux attaques du fort d'Ostie (Lallemend, Boinette 1884 : 251). »
\end{abstract}

\title{
La mise en œuvre lors des sièges
}

Un témoin oculaire du siège de Metz, Bertrand de Salignac, marquis de Fénelon, atteste également du rôle dévolu à Saint-Rémy par le Duc de Guise, à savoir «se pourvoir de bonne heure de touts artifices à feu " (Chabert 1856:10). De manière plus précise, le 7 décembre 1552, face à la menace de l'explosion d'une mine et à l'assaut qui s'ensuivrait, "le seigneur de Sainct Rémy estoit préparé de ses artifices à feu \& engins de guerre, lesquels avoyent esté apportez de bonne heure en une maison prochaine, pour les employer sur les premiers qui viendroye(n)t » (Chabert 1856: 71). Ambroise Paré lui-même, dans son Voyage à Metz, témoigne des préparatifs, mais sans citer l'ingénieur :

«Généralement tous les assiégés délibérèrent de valeureusement se défendre avec toutes machines de guerre :à sçauoir [...] toutes espèces et différences d'artifices de feu, comme boëttes, bariquades,

e-Phaïstos, IX-1 | 2021 
grenades, pots, lances, torches et fusées, cercles entourés de chausses trappes, fagots bruslans ${ }^{45} »$ (Paré 1841 : 705).

\section{Au niveau des effets, Paolo Giovio rapporte
Quint en 1544 (Jiovio $1581: 45^{\mathrm{e}}$ Livre, 640) :}

«D'avantage les François profitoyent grandement avec des pots de terre \& des trompes de bois, getans flammes de feu gluant \& ensouffré, qui s'arrapoyt ${ }^{46}$ contre les corselets \& morrions ». Le côté adhésif du mélange signalé ici est intéressant. D’autres relations de l'usage de ces artifices lors de ce même siège témoignent également de leur cruelle efficacité. Ainsi, «des capitaines [espagnols] furent surpris de cruelles flammes [...]. Au reste, Alvaro Sandeo mesme; ayant les mains \& le visage defigurez de bruleures (Jiovio 1581: $45^{\mathrm{e}}$ Livre, 640, fig.6) ».

Fig.6. Pots et trompes à feu en fonctionnement

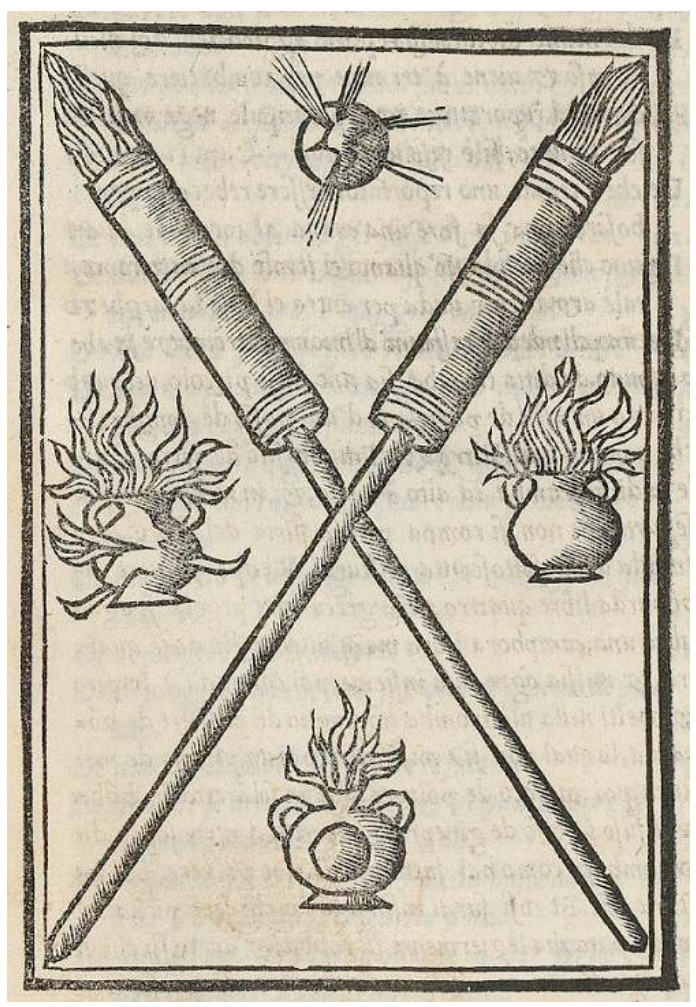

Pour les trompes, si on reprend l'idée d'une longueur totale de 3 à 6 mètres selon les auteurs, la flamme représentée ici ne ferait alors qu'une cinquantaine de $\mathrm{cm}$ de long, tout au plus, soit moins qu'une hallebarde ou même qu'une épée. On ne peut donc que rejoindre Lupicini qui parle d'esquive et de parade facile par l'adversaire.

Della Vale 1529 : n.p. ; https://doi.org/10.3931/e-rara-9770

\section{Conclusion}

Loin des dispositifs farfelus sortis de l'imagination fébrile de théoriciens de la guerre et parfois présentés dans les traités, l'usage des dispositifs décrits ici constitue donc bien une réalité de la guerre au milieu du XVI ${ }^{e}$ siècle, comme ce sera encore le cas lors des 
sièges d'Ypres en 1584 et d'Ostende au début du XVII ${ }^{e}$ siècle (Fino 1977: 226) notamment ; et cela, même si certains dispositifs semblaient assez délicats à mettre en œuvre. Qui plus est, plusieurs d'entre eux ont perduré jusqu'à l'heure actuelle; outre les pots à feu dans lequel on pourrait voir l'ancêtre des bombes au napalm, les grenades, sous différentes formes (défensives, d'exercice, incendiaires, cocktail Molotov, etc.) et les trompes à feu devenues lance-flammes. On constate cependant déjà une évolution entre l'édition de Della Valle, la plus ancienne que nous ayons pu consulter (datée de 1529), et les items collectés par Saint-Rémy, avec l'apparition du chlorure d'ammonium par exemple, mais aussi dans les années qui suivent l'utilisation du $\mathrm{Hg}$ plutôt que du $\mathrm{HgCl}_{2}$, sans doute plus prompt à former HgS. Plus spécifiquement, en ce qui concerne le rôle joué par Saint-Rémy lui-même, cette étude atteste de manière absolue de ses compétences et, d'une façon générale, du niveau de compétence d'un ingénieur français en la matière.

La filiation avec les artifices décrits dans le traité de Della Valle dès 1529 semble incontestable, et prouve, s'il était besoin, du transfert transalpin de certaines technologies. Ces compétences semblent de plus avoir été limitées à une élite, ce qui est conforme à la connaissance que nous pouvons avoir des différents ingénieurs de cette époque et de leur réputation dans le domaine. Enfin, il serait intéressant de réaliser des expériences d'archéologie expérimentale complémentaire pour résoudre définitivement les derniers problèmes pratiques de fabrication et de mises en oeuvre des armements décrits.

\section{BIBLIOGRAPHIE}

ANDRÉ Jacques, « La résine et la poix dans l'antiquité. Technique et terminologie », L'antiquité classique, 33, 1, 1964, p.86-97; https://doi.org/10.3406/antiq.1964.1401

ANONYME, Livre de Canonnerie et artifice de feu, Paris, Vincent Sertenas, 1561 ; URL : https:// gallica.bnf.fr/ark:/12148/bpt6k5421152w.texteImage

AUBIN Nicolas, Dictionnaire de Marine, Amsterdam, Pierre Brunel, 1702 ; URL : https:// gallica.bnf.fr/ark:/12148/bpt6k6281589p.texteImage

BERTHELOT Marcelin, « Les compositions incendiaires dans l'antiquité et au moyen-âge ", Revue des Deux Mondes, 106, 4, août 1891, p.786-822 ; URL : https://www.jstor.org/stable/44761347? seq $=1$

BERTHELOT Marcelin, « Histoire des machines de guerre et des arts mécanique au moyen-âge ", Annales de Chimie et de Physique, $7^{\mathrm{e}}$ série, Tome XIX, 1900, p.289-420 ; URL : https://gallica.bnf.fr/ ark:/12148/bpt6k34919f?rk=42918;4

BIRINGUCCIO Vanoccio, La pyrotechnie, ou L'art du feu : contenant dix livres, ausquels est amplement traicté de toutes sortes et diversité de minières, fusions et séparations des métaux : des formes, moules pour getter artilleries, cloches et toutes autres figures... Traduite d'italien en françois par feu maistre Jaques Vincent, Paris, Claude Frémy, 1572 ; URL : http://www.bvh.univ-tours.fr/Consult/consult.asp? numtable=B372615206_15102\&numfiche $=146 \&$ mode $=3 \&$ offset $=0 \&$ ecran $=0$ 
BOILLOT Joseph, Modelles, artifices de feu et divers instrumens de guerre, avec les moyens de s'en prévaloir pour assiéger, battre, surprendre et deffendre toutes places, Chaumont en Bassig, Q. Mareschal, 1598 ; URL : https://gallica.bnf.fr/ark:/12148/btv1b8610763r/f5.item BOURDEILLE Pierre de, seigneur de Brantôme, Euvres complètes. Tome premier, Paris, Ludovic Lalane, 1864 ; URL : https://gallica.bnf.fr/ark:/12148/bpt6k2063457.texteImage

BOURZAT Jean-Dominique, Lecture contemporaine du Cours de Chymie de Nicolas Lemery, Lyon, Cosmogone, 2005

BOUVET Maurice, «L'huile de Gabian », Revue d'histoire de la pharmacie, 105, 1939, p.5-12 ; URL : https://www.persee.fr/doc/pharm_0035-2349_1939_num_27_105_10771

BRIOIST Pascal, Léonard de Vinci, homme de guerre, Paris, Alma, 2013

BUISSERET David, Ingénieurs et fortifications avant Vauban, Paris, CTHS, 2000

CAGNINA Stefania, Compréhension des mécanismes d'incompatibilité chimique du nitrate d'ammonium par modélisation moléculaire. Thèse de Doctorat, Université P\&M Curie, Paris, 2014 ; URL : http:// www.theses.fr/2014PA066629

CAUVET Désiré, Nouveaux éléments d'histoire naturelle médicale. Tome II, Paris, J. B. Baillière et fils, 1885 ; URL : https://gallica.bnf.fr/ark:/12148/bpt6k771142

CHABERT François-Michel, Journal du siège de Metz en 1552, Metz, Rousseau-Pallez, 1856 ; URL : https://books.google.fr/books/about/Journal_du_siege_de_Metz_en_1552_Documen.html? id=52ZHAAAAYAAJ\&redir_esc=y

CHAMBRIER Paul de, Historique de Péchelbronn-1498-1918, Paris, Attinger Frères, 1919 ; URL : https://iris.univ-lille.fr/handle/1908/4676

CROUY-CHANEL, Emmanuel de, Le canon jusqu'au milieu du XVI e siècle (France, Bretagne et Pays-Bas bourguignons), Volume 1, Thèse pour le doctorat en histoire soutenue le 15-11-2014, Université de Paris I Panthéon-Sorbonne, Paris, 2014

DELLA VALLE Battista, Vallo libro continente appertinentie à Capitanij, retenere \& fortificare una città con bastioni, con novi artificij de fuoco aggionti, Venise, Nicolò d'Aristotile, 1529 ; URL : https:// doi.org/10.3931/e-rara-9770

Du BELLAY Martin et Guillaume, Mémoires. Tome III, Paris, Victor-Louis Bourrilly et Fleury Vindry, 1912 ; URL : https://gallica.bnf.fr/ark:/12148/bpt6k33167v/f262.item

EDEINE Bernard, « Les techniques de fabrication du sel dans les sauneries pré et protohistoriques ainsi que gallo-romaines ", Annales de Bretagne et des pays de l'Ouest, t.82, n¹, 1975, p.1-18 ; DOI : https://doi.org/10.3406/abpo.1975.2757

FINO José-Fédérico, Forteresses de la France médiévale, Paris, A. et J. Picard, 1977

HALLEUX Robert, «Le feu grégeois, ses vecteurs et ses engins de propulsion », in CONTAMINE Philippe, JOUANNA Jacques, ZINK Michel, Colloque la Grèce et la guerre, Paris, Diffusion de Boccard, 2015, p.143-152 ; URL : https://www.aibl.fr/publications/actes-de-colloque/cahiers-de-la-villakerylos-257/article/cahiers-de-la-villa-kerylos-no26

HIME Henry William, Gunpowder and Ammunition, Their Origin and Progress, London, Longmans \& Co, 1904 ; URL : http://www.gutenberg.org/files/54411/54411-h/54411-h.htm\#Page_12

JIOVIO Paolo, Histoire de Paolo Jiovio, Comois, evesque de Nocera, Premier Tome, Paris, Denis Sauvage, 1581 ; URL : https://books.google.fr/books/about/ Histoire_de_Paolo_Jovio_sur_les_choses_f.html?id=RaSkhY-r9G8C\&redir_esc=y 
LALLEMEND Marcel, BOINETTE Alfred, Jean Errard de Bar-le-Duc, sa vie, ses œuvres, sa fortification, Paris, Thorin et Dumoulin, 1884 ; URL : https://gallica.bnf.fr/ark:/12148/ bpt6k65723282.texteImage

MARICHAL Paul, Catalogue des actes de François I ${ }^{\text {er }}$, Tome 8, Paris, Imprimerie Nationale, 1905 ; URL : https://gallica.bnf.fr/ark:/12148/bpt6k5720308k?rk=21459;2\#

MENANT François, « Pour une histoire médiévale de l'entreprise minière en Lombardie », Annales. Économies, sociétés, civilisations, t.42, n4, 1987, p.779-796; URL : https://doi.org/10.3406/ahess. 1987.283419

PARÉ Ambroise, CEuvres complètes d'Ambroise Paré - Tome 3, Paris, Malgaigne, J.-F., 1841 ; URL : https://gallica.bnf.fr/ark:/12148/bpt6k61149766.texteImage

PAUCOT Hugues, FAUCHERRE Nicolas, « Saint-Paul-de-Vence ou Jean de Saint-Rémy à l'école des Sangallo », in FAUCHERRE Nicolas, MARTENS Pieter, PAUCOT Hugues, La genèse du système bastionné en Europe 1500-1550, Navarrenx, Cercle Historique de l'Arribère, 2014, p.151-164

PELOUZE Edmond, Traité de l'éclairage au gaz tiré de la houille, des bitumes, des lignites, de la tourbe, des huiles, des résines, des graisses, Paris, Maison ; URL : 1839, https://iris.univ-lille.fr/handle/ 1908/5185

PETITOT Claude Bernard, Collection complète des mémoires relatives à l'histoire de France, Tome XXVII. Mémoires de la vie du Maréchal de Vieilleville. Paris, Foucault, 1822 ; URL : https://gallica.bnf.fr/ark:/ $12148 /$

bpt6k36381p.r=M\%C3\%A9moires\%20de\%20la\%20vie\%20du\%20Mar\%C3\%A9chal\%20de\%20Vieilleville? rk=21459;2

REINAUD Joseph Toussaint, FAVE Ildefonse, Histoire de l'artillerie, $1^{\text {ère }}$ partie. Paris, J.Dumaine, 1845 ; URL : https://books.google.fr/books/about/ Histoire_de_1_Artillerie_1re_partie_Du_f.html?id=6jZkAAAAcAAJ\&redir_esc=y

REINAUD Joseph Toussaint, FAVE Ildefonse, « Du feu grégeois, des feux de guerre et des origines de la poudre à canon ", Journal Asiatique, XIV, octobre 1849, p.257-327 ; URL : https:// gallica.bnf.fr/ark:/12148/bpt6k93154r/f257.item

REN Yafeng, ZHU Jianxin, « Leaching Behavior and Risk Control of Waste Mercury Chloride Catalyst Through Mechanochemical Reaction Using Sulfur », Environ. Engineering Science, t.32, $\mathrm{n}^{\circ} 9$, 2015, p.816-823 ; DOI : https://doi.org/10.1089/ees.2015.0014

SAINT REMY Surirey de, Mémoires d'artillerie. Tome I, La Haye, Jean Néaulme, 1741 ; URL : https:// gallica.bnf.fr/ark:/12148/bpt6k62773351.texteImage

SIEMIENOWICZ Casimir, Grand art d'artillerie... mise en françois par Pierre Noizet. Amsterdam, J. Jansson, 1651; URL : https://gallica.bnf.fr/ark:/12148/bpt6k6308069d/f15.item.texteImage

SMITH Kay Douglas, « From the Dung Heap to the Stars: a History of Early Gunpowder », BDHS Journal, 23, September 2018, p.37-40; URL : http://btckstorage.blob.core.windows.net/site15733/ BDHSJ\%20No.23\%20September\%202018.pdf

UFANO Diego, Artillerie, c'est à dire vraye instruction de l'artillerie et de toutes ses appartenances. Zutphen, André D’Aelst, 1621 ; URL : https://books.google.fr/books/about/ Artillerie_c_est_a_dire_Vraye_instructio.html?id=ZXaJH6qnJf8C\&redir_esc=y

VENTURI Jean-Baptiste, Essai sur les ouvrages physico-mathématiques de Léonard de Vinci, Paris, Duprat, 1797 ; URL : https://doi.org/10.3931/e-rara-61985

VÉRIN Hélène, La Gloire des Ingénieurs, Paris, Albin Michel, 1993 


\section{NOTES}

1. Auteur du «Liber Ignium » daté du XIII ${ }^{\mathrm{e}}$ siècle.

2. Citons par exemple Halleux 2015.

3. Défense, protection (Pour l'essentiel, tous les termes anciens sont explicités sur deux sites de références, https://www.cnrtl.fr/definition/portail et www.atilf.fr/dmf, sites consultés au cours des mois de janvier et de février 2021).

4. BNF, Manuscrits, Clairambault 345, fo 95-112 en ligne sur Gallica, site consulté le 15/01/2021.

5. BNF, Manuscrits, Clairambault 346, $\mathrm{f}^{\circ} 69 \mathrm{r}^{\circ}$ et $\mathrm{v}^{\circ}$ en ligne sur Gallica, site consulté le 15/01/2021. Ce document avait déjà été signalé par Hélène Vérin (Vérin 1993 : 120 et 414).

6. L'orthographe du texte d'origine a été respectée.

7. Aiguille.

8. Clous de grande longueur.

9. Archal : Fil de fer (Fe) ou de laiton (alliage de cuivre et de zinc).

10. Le fer-blanc est une tôle relativement fine d'acier doux ou extra doux (teneur en carbone inférieure à $0,08 \%$ ), recouverte d'étain sur les deux faces. L'invention du fer-blanc remonte au $\mathrm{XIII}^{\mathrm{e}}$ siècle et est attribuée aux métallurgistes de Bohême et de Saxe. Le secret de sa fabrication fut longtemps et précieusement conservé, et la première manufacture ne sera fondée en France qu'en 1665 (fr.wikipedia.org/wiki/Fer-blanc, site consulté le 08/01/2021).

11. L'oing était un onguent, constitué de graisse, généralement de porc, parfois synonyme de suif. Il en sera question ultérieurement sous le nom de " gresse de pourceau ».

12. L'huile de lin est une huile végétale extraite des graines mûres du lin cultivé, pressées à froid et/ou à chaud, constituée à plus de $70 \%$ d'acides gras polyinsaturés. Elle polymérise spontanément à l'air, avec une réaction exothermique, ce qui la rend inflammable sous certaines conditions.

13. L'huile d'olive est une variété d'huile alimentaire, à base de matière grasse végétale extraite des olives, et composée d'environ $99 \%$ de triglycérides.

14. Goudron.

15. Voir à ce sujet (André 1964).

16. Qui consistait essentiellement à en éliminer l'eau et à lui faire subir une clarification.

17. fr.wikipedia.org/wiki/Colophane, site consulté le 08/01/2021.

18. https://www.ilo.org/dyn/icsc/showcard.display?p_lang=fr\&p_card_id=1021\&p_version=2 site, consulté le 03/02/2021.

19. Responsable entre autres des explosions d'AZF à Toulouse en septembre 2001 et du port de Beyrouth en août 2020.

20. Poudre non battue ou émiettée, moins puissante que la poudre employée pour les couleuvrines (De Crouy-Chanel $2014: 61$ ).

21. « ... polvere de bo $(m) b a r d a$ parte doe, pezza greca overo pegola spagna parte une, pegola navale, over pegola de nave parte una pista sottilissimamente ogni cosa insieme in un mortaro, poi habbi treme(n)tina alla satisfatione in una caldara, \& mettila sopra il fuoco \& metti dentro alquantao olio di lino, no(n) potendo haver olio de ginepro » (Della Valle 1529 : non paginé, traduction de l'auteur).

22. Libra, la livre poids.

23. Nitrate de potassium (salpêtre).

24. Tartre : tartrate acide de potassium (Bourzat $2005: 223$ ).

25. Sarcocolle : résine du sarcocollier.

26. «Peghe " : poix.

27. « Sal coctile » : sel cuit. Berthelot a cru y reconnaître le salpêtre (Berthelot $1891: 811$ ), mais il s'agirait plus vraisemblablement de chlorure de sodium obtenu par ébullition et évaporation de l'eau (Edeine $1975: 7)$. Selon Hime (1904: 13), aucune mention de salpêtre n'a été trouvée avant le XIII ${ }^{\text {e }}$ siècle ; il ne pouvait donc pas rentrer dans la composition des feux grégeois. On pourrait 
faire la même remarque au sujet de la composition donnée par Léonard décrite par Venturi. Reinaud et Favé confirment que « rien n'autorise à traduire ainsi [c'est-à-dire salpêtre]; le sel ordinaire a été souvent employé dans les artifices» (1849: 283). D'après Halleux (2015: 149) la présence du $\mathrm{NaCl}$ produit une flamme plus chaude. En réalité, $\mathrm{NaCl}$ pouvait aussi contribuer à l'instabilité du nitrate d'ammonium comme dit précédemment.

28. Huile de pétrole.

29. Huile commune : sans doute de production locale (lin, olive...).

30. Gomme du tragacanthe. Il s'agit d'un mélange de polysaccharides qui se compose principalement de tragacanthine (60-70\%) et de bassorine (30-40\%). Au contact de l'eau, la tragacanthine se dissout pour former un hydrosol colloïdal, tandis que la bassorine insoluble gonfle pour former un gel. On obtient ainsi une pâte adhésive. (https://fr.wikipedia.org/wiki/ Gomme_adragante, site consulté le 19/01/2021).

31. Bien que l'envoi vers l'adversaire par l'artillerie névrobalistique ait été longtemps en usage.

32. Aujourd'hui Székesfehérvár en Hongrie.

33. Verre.

34. $\mathrm{NaCl}$.

35. Pétrole.

36. « ...habbi parte cinque di polverere di artellaria grossa, parte tre de salmistro refinato, parte dua de Solfore, parte una di Rasapina, parte mezza di Camphora, parte mezza di Trementini, parte mezza di vitro pisto, no(n) troppo, parte mezza di Sal co(m)mune, parte mezza di Oglio de sasso, \& oglio di lino ana ciò̀ equale parte, \& parte mezza de acqua vita, \& tutte queste cose mescolando insieme molto be(n) forte, da poi piglia una pezza de tela quanto voi fare la palla, \& falla à modo d'una borsa, \& impila di detta mistura " (Della valle 1529: $10 \mathrm{v}^{\circ}$, traduction de l'auteur).

37. BNF, Clairambault $345 \mathrm{f}^{\circ} 103$.

38. Unité de longueur égale à douze pouces, soit approximativement $33 \mathrm{~cm}$.

39. Mesure de longueur (égale à la largeur - ou la longueur - de la main, un peu plus de $20 \mathrm{~cm}$ ).

40. B.N.F, Clairambault $345 f^{\circ} 103$.

41. La partie des Alpes comprise dans les provinces de Brescia et de Bergame a constitué au XX siècle un des principaux centres italiens d'activités minières et métallurgiques. Au Moyen-âge, c'est même vraisemblablement le plus important de ces centres et il atteint son apogée pendant les premiers temps de la domination vénitienne. C'est ici qu'est accompli au XIII ${ }^{\mathrm{e}}$ siècle un progrès décisif de la technique métallurgique avec le passage du procédé direct de traitement du minerai de fer au procédé indirect ; les vallées lombardes conserveront de ce fait pendant deux siècles une avance technologique considérable sur le reste de Europe (Menant 1987: 779). Le métal dont étaient cerclées les bombardes a notamment été qualifié de "fer lombard ».

42. Brasse : mesure de longueur équivalant environ à la longueur des bras étendus, soit environ 1,60 mètre environ, et donc ici 2,4 mètres.

43. Le sciage devait sans doute permettre de l'évider, ce qui aurait été difficile à faire par alésage vu la longueur de la pièce, de recouvrir l'intérieur de l'âme avec du métal et facilitait le remplissage.

44. Sous-produit fibreux non tissé issu essentiellement du travail du chanvre ou du lin, qui pouvait servir éventuellement d'agent de texture dont l'enchevêtrement constituait alors l'armature de la charge incendiaire et l'empêchait de se désagréger (Halleux 2015 : 146) ; dans ce cas précis, il est utilisé ici seulement pour éviter que le mélange de réactifs ne puisse tomber de la lance lors des manipulations.

45. La notion de fusée est intéressante. Cependant, aucun des manuscrits cités n'y fait allusion.

46. «s'accrocher». 


\section{RÉSUMÉS}

Si divers traités tels celui de Della Valle, imprimé en 1521, jusqu'à celui de Boillot édité à la fin du même siècle, décrivent les « artifices » incendiaires, voire explosifs, utilisés lors des guerres de siège au XVI ${ }^{\mathrm{e}}$ siècle, aucune étude de leur fabrication, de leur mode de fonctionnement et de leur mise en œuvre n'a jamais vraiment été entreprise. En se basant sur l'exemple du siège de Metz en 1552 et l'analyse de documents manuscrits originaux fournissant des informations détaillées sur les matériaux et les matières entrant dans leur composition, puis en utilisant les descriptifs donnés par les traités, il devient possible d'établir l'état de l'art des « cercles ", « pots » ou encore " trompes à feu ». Des recherches sur les différents mécanismes physico-chimiques fondamentaux mis en œuvre pour favoriser inflammabilité et explosivité, permettent d'en appréhender toute l'efficacité. Enfin, divers témoignages d'époque nous éclairent sur les effets de ce type d'armement.

Although various treaties such as that of Della Valle, printed in 1521, or that of Boillot published at the end of the same century, describe the incendiary, even explosive devices used during the siege wars of the sixteenth century, no study of their manufacture, mode of operation or implementation has ever really been undertaken. Based on the example of the siege of Metz in 1552 and the analysis of original handwritten documents providing detailed information concerning the materials used in their composition, and applying the descriptions provided in the treaties, it becomes possible to establish the state-of-the-art of "circles", "pots" or even "fire lances". Research on the various fundamental physicochemical mechanisms implemented to promote flammability and explosiveness allows us to understand their full effectiveness. Finally, various testimonies of the time shed light on the effects of this type of armament.

\section{INDEX}

Mots-clés : histoire des techniques, armement, explosif, guerre, siège

Keywords : history of technology, weapon, warfare, firework, fire device

\section{AUTEUR}

\section{HUGUES PAUCOT}

Titulaire d'une maîtrise en chimie et d'un doctorat en Sciences de la Terre et de l'Environnement de l'Université Libre de Bruxelles, actuellement ingénieur de recherche à l'Université de Pau et des Pays de l'Adour (UPPA), j'ai entamé une thèse en Histoire Moderne au sein du laboratoire ITEM (EA 3002) qui porte sur la vie et l'œuvre des ingénieurs militaires italiens en France au XVI ${ }^{\mathrm{e}}$ siècle. Auteur de plusieurs articles sur les fortifications des villes de Navarrenx et de Saint-Paulde-Vence, et coéditeur de « La genèse du système bastionné en Europe 1500-1550 ». 